\title{
FUZZY INFERENCE SYSTEM \\ FOR THE IDENTIFICATION OF OVER-THE-COUNTER (OTC) DRUGS
}

\author{
Eduardo E. Zurek ${ }^{* *}$ \\ Daniel J. Romero ${ }^{* * *}$ \\ Hernán D. Blanco \\ Carlos A. Gutiérrez ${ }^{* *+* *}$ \\ Recibido: $10 / 04 / 2012$ \\ Aceptado: 07/05/2013
}

\begin{abstract}
This document shows the details of the implementation of a fuzzy inference system, for the identification of four over-the-counter drugs (Naproxen, Calcium Carbonate, Muvett and Winadol), by using a Raman Spectroscopy, which output is the characterization of the substance. Data obtained from Raman Spectroscopy are modeled with Matlab®- Fuzzy Logic Toolbox.
\end{abstract}

Key words: Fuzzy logic, Raman spectroscopy, Artificial Intelligence, Soft Computing, Signal Processing.

Artículo de investigación científica y tecnológica.

** Profesor investigador. Grupo de Investigación en Robótica y Sistemas Inteligentes, Departamento de Ingeniería de Sistemas, Universidad del Norte, Barranquilla, Colombia. Correo: ezurek@uninorte.edu.co

*** Estudiante del Programa de Ingeniería de Sistemas, Universidad del Norte, Barranquilla, Colombia. Correo: djromer@gmail.com

**** Estudiante del Programa de Ingeniería de Sistemas, Universidad del Norte, Barranquilla, Colombia. Correo: hdblanco.m@gmail.com

***** Profesor investigador. Grupo de Investigación en Tecnologías de Ingeniería Naval - GITIN. Programa de Electrónica. Escuela Naval de Suboficiales ARC Barranquilla, ENSB. Barranquilla, Colombia. E-mail: ing.cagm@gmail.com. 


\section{SISTEMA DE INFERENCIA DIFUSA PARA LA IDENTIFICACIÓN DE MEDICAMENTOS COMERCIALES}

\section{RESUMEN}

Este documento muestra los detalles de la implementación de un sistema de inferencia difusa para la identificación de medicamentos comerciales (Naproxeno, Carbonato de Calcio, Muvettt y Winadol), aplicando Espectroscopia Raman, cuyo resultado es la caracterización de la sustancia. Los datos obtenidos con Espectroscopia Raman se modelan con Matlab ${ }^{\circledR}$ - Fuzzy Logic Toolbox.

Palabras clave: lógica fuzzy, espectroscopia raman, inteligencia artificial, computación suave, procesamiento de señales. 


\section{INTRODUCTION}

Raman spectroscopy has experienced a big development in recent years, to the point of being utilized as a portable way for the identification of unique characteristics in some substances, due to the advantage provided by Raman Spectroscopy of analyzing the substance with only a little sample of this one. As an example of the current work, it has been considered the detection of contaminating agents in water applying the Raman spectroscopy [1]. A rapid detection protocol suitable for use by first-responders to detect anthrax spores using a low-cost, battery-powered, portable Raman Spectroscopy has been developed as well [2].

Fuzzy logic provides a natural method for the construction of systems to emulate human decision taking processes. Literature on the subject of fuzzy logic systems (FLSs) is extensive and applications, particularly in the field of fuzzy control and fuzzy expert systems, are prevalent. In essence, fuzzy set theory and fuzzy logic are a generalization of crisp set theory and traditional, dual-valued $\operatorname{logic}[3]$.

A fuzzy set $F$ of a universe of discourse $U$ is characterized by a membership function $\mu_{\mathrm{F}}: U \rightarrow$ $[0,1]$ which associates with each element $u$ of $U$ a number $\mu_{\mathrm{F}}(\mathrm{u})$ in the interval $[0,1]$ which represents the grade of membership of $\mathrm{u}$ in $\mathrm{F}$. The label $\mathrm{F}$ of a fuzzy set is often some linguistic term like "small," "large," etc. A linguistic variable is a variable whose values are words in a natural or artificial language, and these words are labels of fuzzy sets [4].

The Fuzzy inference system is a method which interprets the values in the input vector and, based on user defined rules, assigns values to the output vector [5], this system is a popular computing framework based on the concepts of fuzzy set theory, if-then rules, and fuzzy reasoning [6]. As an example this system has led to significant advances in medicine, where it was implemented a fuzzy model for computation of margins in cancer treatment [7], and had made possible to develop another Fuzzy Interference System (FIS) to identify substances characterized on the Raman spectroscopy. These substances are: Calcium carbonate, shown in Fig. 1 (May be available in countries like Colombia and Portugal); Muvett, shown in Fig. 2 (Trimebutine, may be available in the countries: Ecuador and Colombia); Winadol, shown in Fig. 3 (Paracetamol, is available and Found in Colombia); Naproxen, shown in Fig. 4 (Naproxen, is in a group of drugs called non-steroidal anti-inflammatory drugs, and available in the majority of world). The generic names of these OTC drugs can be found in [8].

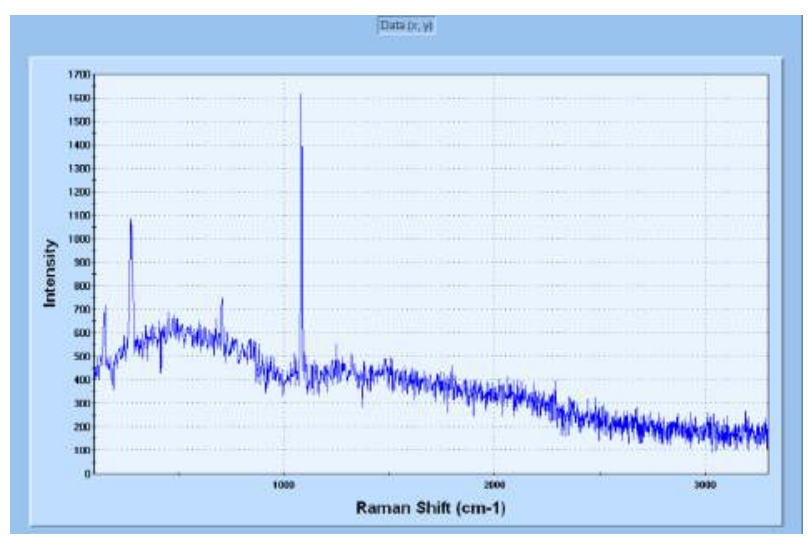

Fig. 1: Calcium carbonate's Raman spectroscopy output chart

Source: the authors

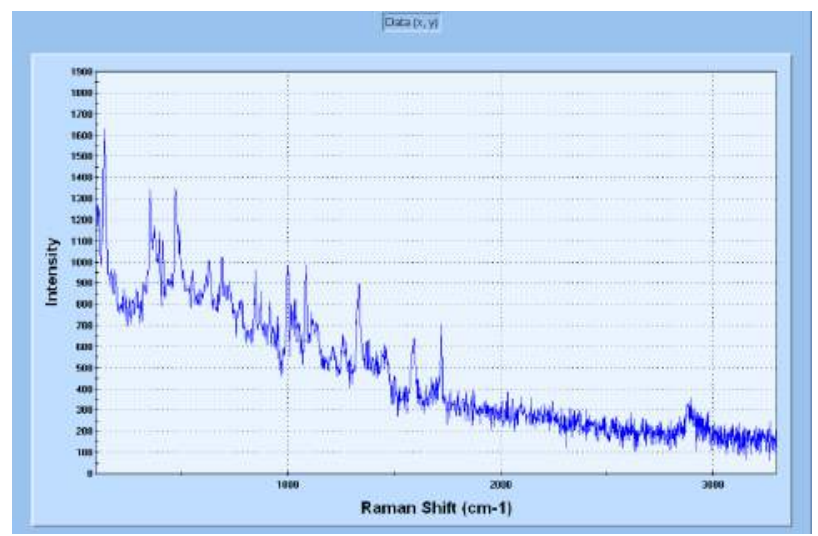

Fig. 2: Muvett's Raman spectroscopy output chart. Source: the authors 


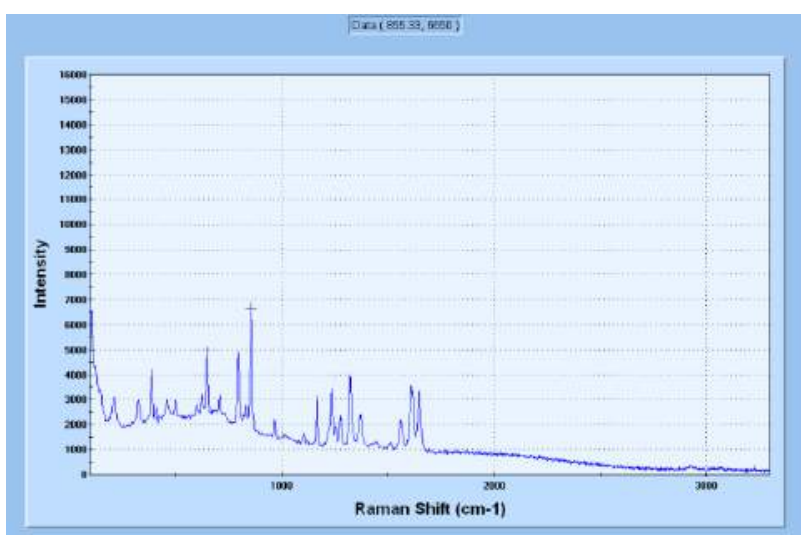

Fig. 3: Winadol's Raman spectroscopy output chart. Source: the authors

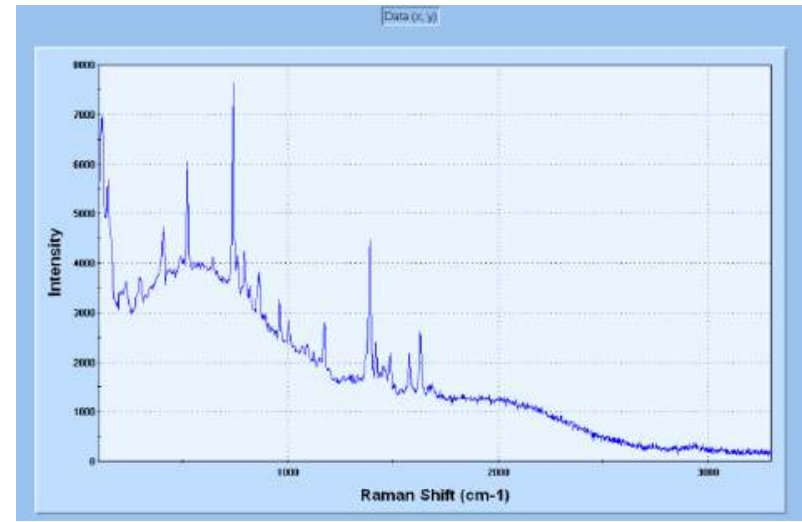

Fig. 4: Naproxen's Raman spectroscopy output chart. Source: the authors

\section{PROPOSED APPROACH}

The spectra samples were taken using a Raman spectroscopy EZRaman-M Field Portable Series with spectral resolution $6 \mathrm{~cm}^{-1}$. [9], for taking the samples needed in order to obtain characteristic data of drugs. These samples were taken in two occasions with different objectives, first of all for the establishment of medicine taken on account within this project, and secondly for the simulation of an error in manufacturing process, by altering the initial composition of tests, mixing them with different substances.

The tests described above were performed with the same exposure tolerance in Raman spectroscopy, which was Integration (s): 12, Average: 6 and Boxcar: 1 , the parameters determine the efficiency in making the spectrum of the substance, and the assignment of these values was the result of the search for that efficiency.

Data obtained via Raman spectroscopy had to be regularized, due to the fact that they are not taken in an ideal ambient and thus contain noise signals. In order to avoid having those filtered data, we chose the more significant peaks of the substance and compared them with existing results within a database of InPhotonics .inc, named "Forensic Raman Spectral Database" [10], in order to be completely sure that output data from the spectroscopy were similar to those obtained in previous results.

After completing test with Raman spectroscopy, next step was the implementation for a Fuzzy inference system using Matlab@-Fuzzy Logic Toolbox-. We built the rules set, defined the membership functions, and analyzed the behavior of a fuzzy inference system (FIS) [11].

In our FIS implementation we used Gaussian membership functions as input [12], as evidenced in Fig. 5, and for output triangular shaped membership functions [13], as it is shown in Fig. 6. After data input evaluation, the system would choose an option within the range of the output membership function.

\section{FISOTC PROCESS}

\section{a. Data selection algorithm}

Raman spectroscopy shows a file which has the result of the substance exposed, this result is composed of wave number shift and intensity, corresponding to the $\mathrm{x}$ axis and the $\mathrm{y}$ axis respectively. In this file (.xls) the most significant values associated with the y axis are filtered, and then ranked from highest to lowest to select the top 10 values. Finally values associated with the $\mathrm{x}$ axis are sorted from lowest to highest.

This procedure is done in order to obtain the data and significant characteristic of the exposed sample in Raman spectroscopy. 


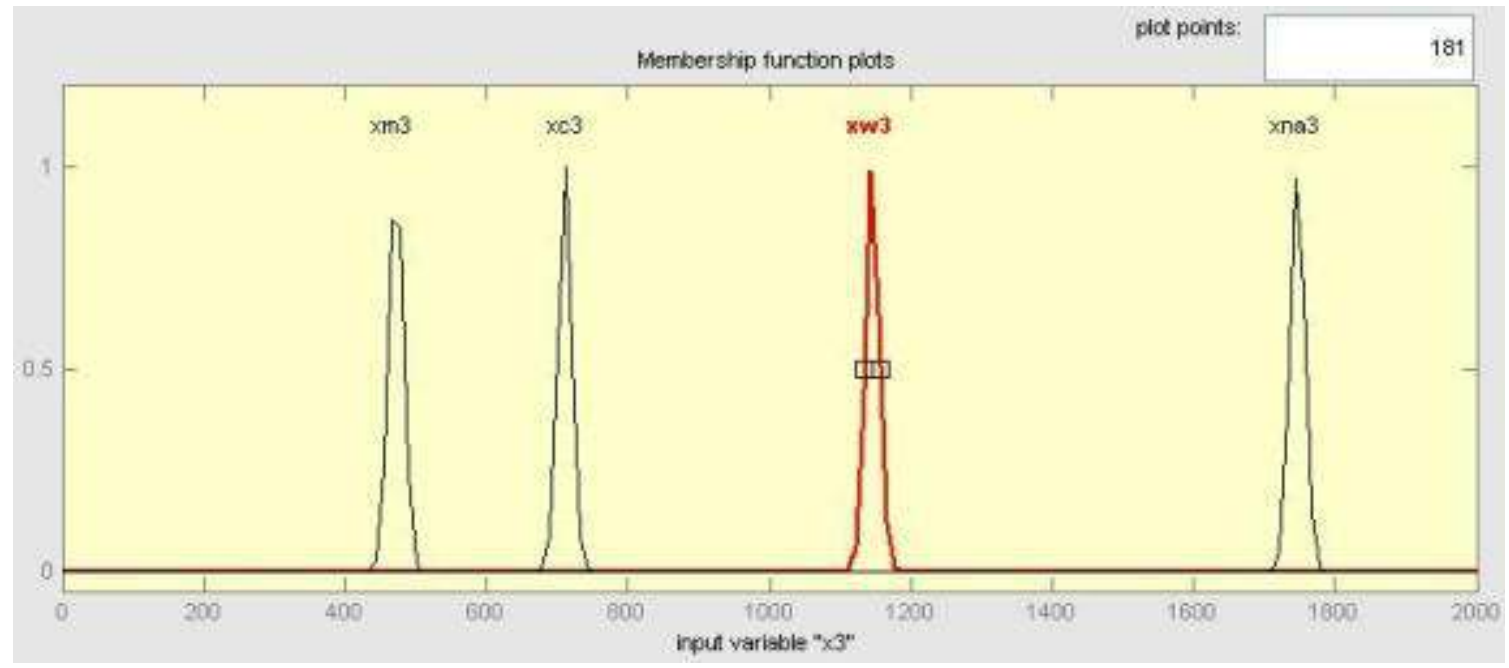

Fig. 5: Membership function input.

Source: the authors

This procedure is done in order to obtain the data and significant characteristic of the exposed sample in Raman spectroscopy.

\section{b. Fuzzy inference process}

We evaluated in Fuzzy Logic Toolbox ten entries associated with a numeric value which is within a domain $(0,2000)$, this guarantees the inclusion of the significant values of the sample chosen by the previous algorithm. We also used a Gaussian membership function for the inputs which will de- termine the value of membership which is bounded between 0 and 1 .

Each entry has associated five membership functions, where each function corresponds to a peak of the substance to be compared.

The first entry is compared with the peak having the smallest wavelength in the spectrum of winadol, with the peak having the smallest wavelength in the spectrum of calcium, with the peak having the smallest wavelength in the spectrum the naproxen and, with the peak hav-

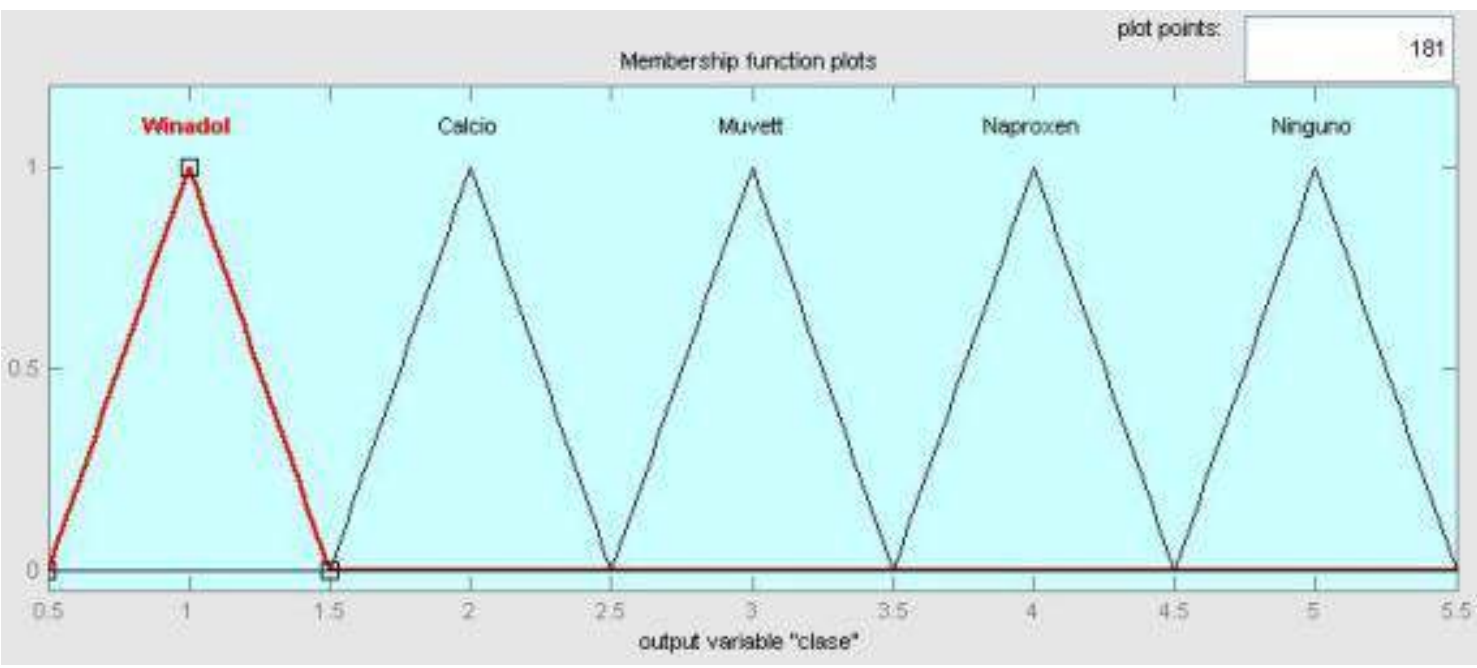

Fig. 6: Membership function output.

Source: the authors 
ing the smallest wavelength in the spectrum of muvett.

The second entry is compared with the peak having the second lowest wavelength in the spectrum of winadol, with the peak having the second lowest wavelength in the spectrum of calcium, with the peak having the second lowest wavelength in the spectrum of naproxen, with the peak having the second lowest wavelength in the spectrum of muvett.

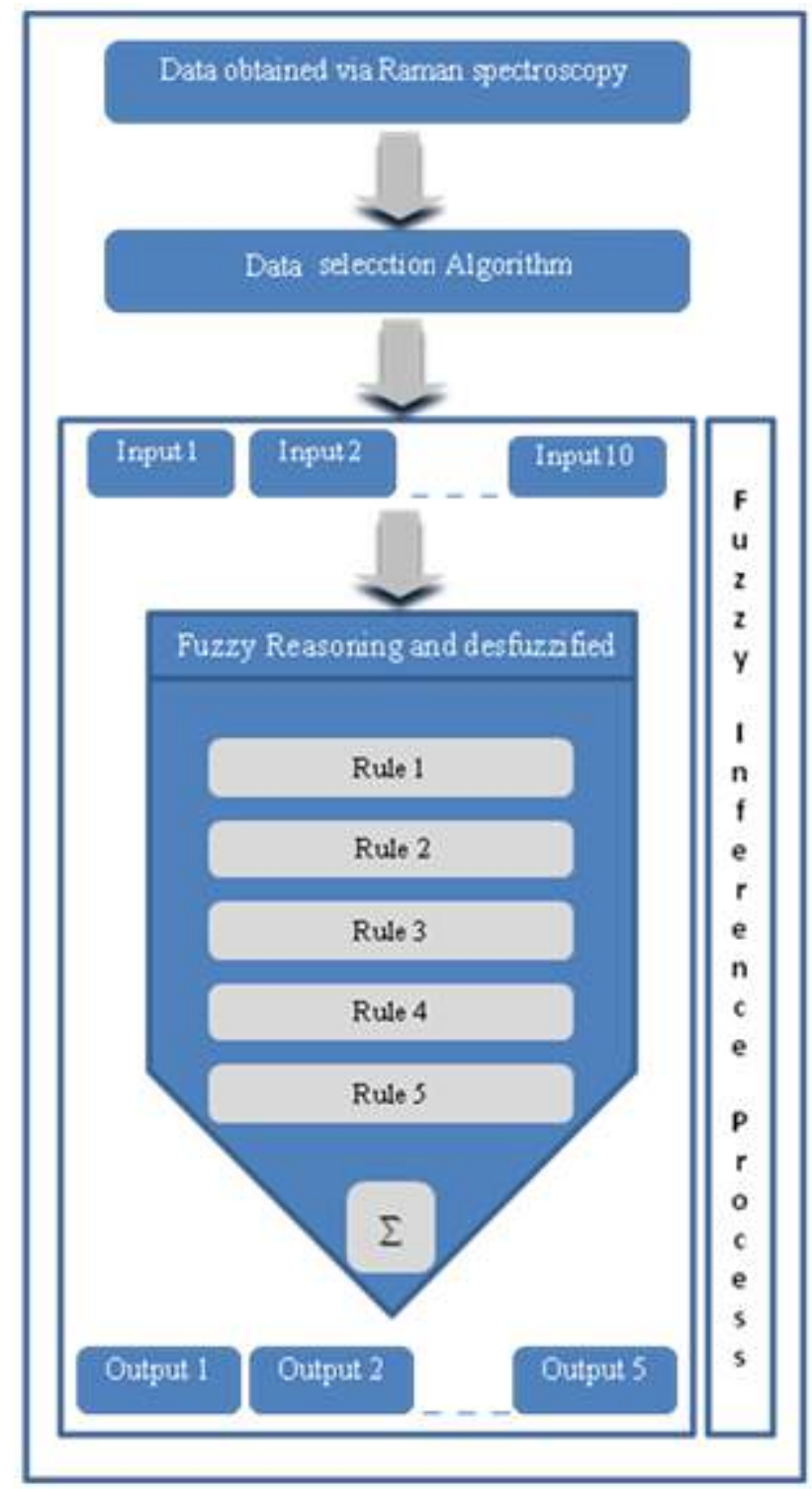

Fig. 7: Fisotc Process
The same procedure applies to all entries until the tenth is compared with the tenth peak wavelength in the spectrum of winadol, with the tenth peak wavelength in the spectrum of calcium, with the tenth peak wavelength in the spectrum of naproxen, with the tenth peak wavelength in the spectrum of muvett.

With inputs already defined, we turn to fuzzy Reasoning and desfuzzified where first we create five rules taking into account that in a fuzzy inference system, a rule consists of an antecedent, a consequent and an operator ("and”, "or ").

The rules depend on input values which are within a number of Different linguistic fuzzy sets. [14].

For our FIS the first 4 rules are associated with the medications to recognize and the fifth rule is associated with an unknown outcome of the first four rules.

The rules are activated as follows:

Rule 1. Winadol, it activates if the first entry corresponds to the first peak of winadol and the second with the second peak winadol and the third with the third peak of winadol. This process continues until the tenth entry is compared with the tenth winadol peak.

Rule 2. Calcium, it activates if the first entry corresponds with the first peak of calcium and the second with the second peak of calcium and the third with the third peak of calcium. This process continues until the tenth entry is compared with the tenth calcium peak.

Rule 3. Naproxen, it activates if the first entry corresponds to the first peak of naproxen and the second with the second peak of naproxen and the third with the third peak of naproxen, this process continues until the tenth entry is compared with the tenth naproxen peak.

Rule 4. Muvett, it activates if the first entry corresponds to the first peak of muvett and the second with the second peak muvett and the third with 
the third peak of muvett, this process continues until the tenth entry is compared with the tenth muvett peak.

Rule 5. The none, this is triggered if the first entry corresponds with the first peak of the none, and the second with the second peak of the none, and the third with the third peak of the none, this process continues until the tenth entry is compared with the tenth peak of the none.

The outputs are activated with a triangular desfuzzified $(\Sigma)$ for each of the substances in a sample space from 0.5 to 5.5 , where the winadol is centered in 1 , calcium is centered on 2 , muvett is centered on 3, the naproxen is centered on 4 and the none is centered on 5, as shown in Fig. 6.

The result of this process is the identification of substances exposed in Raman spectroscopy, which is reflected in the program (Fisotc) developed for this process, Fisotc receives a .xls file where the spectrum of the substance supplied is located. After Raman spectroscopy exposure, Fisotc outputs the name and graphics of the substance recognized by the fuzzy inference system.

In Fig 8, we can see an interface where the program reveals that the spectrum file entered belongs to the drug Winadol.

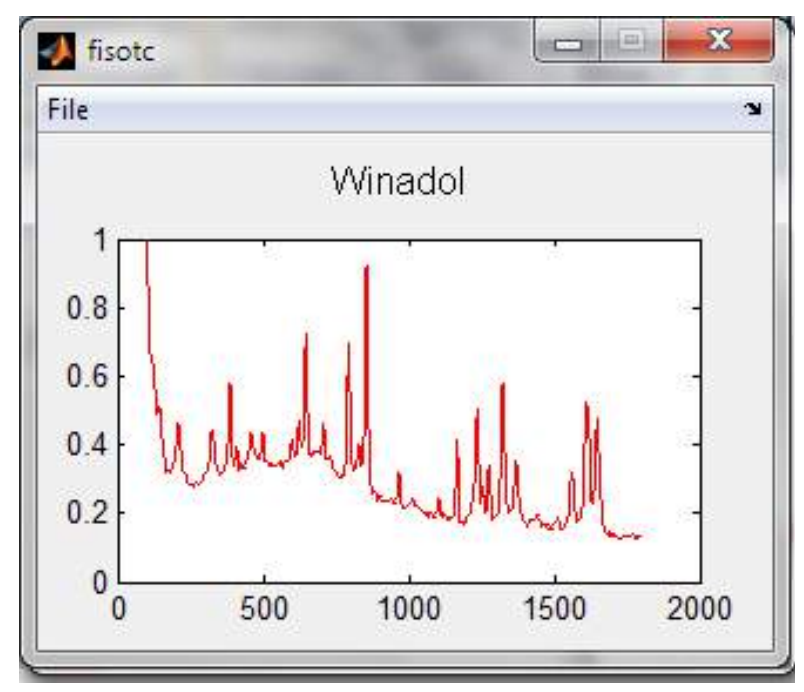

Fig. 8: Fisotc resulted.

\section{CONCLUSION}

As a result of this process, we developed a tool which can be trained to identify any substances capable to be analyzed with Raman spectroscopy. The process here was tested with the following medications: Naproxen, Calcium Carbonate, Muvett and Winadol, so the Fuzzy Inference System received as input the data obtained from Raman spectroscopy, and the output is substance that is more related to the sample, if it is one of the medications mentioned above, else, the system displays a message indicating that the spectrum is unknown.

It should be noted that all tests that were analyzed with generic samples were validated in previous research experiences, also the system is designed to recognize a greater number of medications than the one used before, opening the possibility to create a database with different drug samples before being used by Fisotc.

\section{REFERENCES}

[1] Gang Li; Minghong Chen; Tianyun Wei, "Application of Raman Spectroscopy to Detecting Organic Contaminant in Water", Control, Automation and Systems Engineering, 2009. CASE 2009. IITA International Conference on, 493 - 495, 11-12 July 2009.

[2] X. Zhang et al, "Rapid detection of an anthrax biomarker by surface-enhanced Raman spectroscopy," J. Am. Chem. Soc 127, no. 12 (2005): 4484-4489.

[3] Jang, Sun \&Mizutani, "Neuro-Fuzzy and Soft Computing: A Computational Approach to Learning and Machine Intelligence". Upper Saddle River, N.J.: Prentice-Hall, 1997002E.

[4] Li-Xin Wang, "Design and analysis of fuzzy identifiers of nonlinear dynamic systems," Automatic Control, IEEE Transactions on 40, no. 1 (1995): 11-23.

[5] Matlab®, "Fuzzy Logic Toolbox 2.1" at http://www. mathworks.fr/academia/student_version/r2009a_ products/fuzzylogic.pdf, 2010.

[6] D. Ramot et al., "Complex fuzzy logic," Fuzzy Systems, IEEE Transactions on 11, no. 4 (2003): 450-461.

[7] B. Mzenda et al., "Implementation of a fuzzy model for computation of margins in cancer treatment," in Fuzzy

Source: the authors 
Systems (FUZZ), 2010 IEEE International Conference on, 2010, 1-8.

[8] "Drugs.com / Prescription Drugs - Information, Interactions \& Side Effects," http://www.drugs.com, 2010.

[9] EnwaveOptronics, Inc, "EZRAMAN-M Field Portable Series PORTABLE RAMAN ANALYZERS" at http:// www.enwaveopt.com/doc/EZRaman-MFieldPortableSeries.pdf, 2010.

[10] InPhotonics, Inc.,"Forensic Raman Spectral Database". Version 1.0, 2000.

[11] O. Castillo and P. Melin, Soft computing and fractal theory for intelligent manufacturing (Springer, 2003).
[12] "Gaussian curve built-in membership function MATLAB,"n.d,http://www.mathworks.com/help/ toolbox/fuzzy/gaussmf.html, 2010.

[13] "Triangular-shaped built-in membership function, MATLAB" n.d, http://www.mathworks.com/help/ toolbox/fuzzy/trimf.html, 2010.

[14] Matlab®, "Fuzzy Logic Toolbox 2.1" at http://www. mathworks.com/help/toolbox/fuzzy/fp351dup8.html, 2010. 